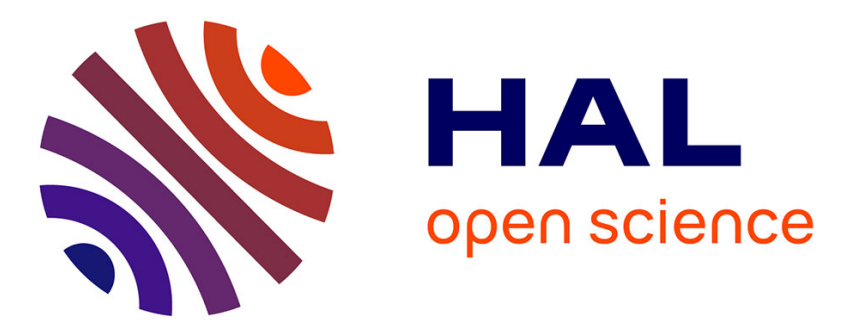

\title{
Reflection function of a plane sound wave in a cylindrical tube
}

J.D. Polack, X. Meynial, J. Kergomard, C. Cosnard, M. Bruneau

\section{To cite this version:}

J.D. Polack, X. Meynial, J. Kergomard, C. Cosnard, M. Bruneau. Reflection function of a plane sound wave in a cylindrical tube. Revue de Physique Appliquée, 1987, 22 (5), pp.331-337. 10.1051/rphysap:01987002205033100 . jpa-00245548

\section{HAL Id: jpa-00245548 https://hal.science/jpa-00245548}

Submitted on 1 Jan 1987

HAL is a multi-disciplinary open access archive for the deposit and dissemination of scientific research documents, whether they are published or not. The documents may come from teaching and research institutions in France or abroad, or from public or private research centers.
L'archive ouverte pluridisciplinaire HAL, est destinée au dépôt et à la diffusion de documents scientifiques de niveau recherche, publiés ou non, émanant des établissements d'enseignement et de recherche français ou étrangers, des laboratoires publics ou privés. 


\title{
Reflection function of a plane sound wave in a cylindrical tube
}

\author{
J. D. Polack, X. Meynial, J. Kergomard, C. Cosnard and M. Bruneau \\ Laboratoire d'Acoustique, U.A.-C.N.R.S. 1101, BP 535, 72017 Le Mans Cedex, France
}

(Reçu le 12 novembre 1986, accepté le 26 janvier 1987)

\begin{abstract}
Résumé. - La dissipation et la dispersion d'ondes planes dans un tuyau cylindrique étant proportionnelle à la racine carrée de la fréquence, la forme analytique de la réponse impulsionnelle existe. Nous étudions la fonction de réflexion à l'entrée d'un tuyau, c'est-à-dire la transformée de Fourier inverse du coefficient de réflexion, et particulièrement les importantes distorsions dues au traitement numérique ainsi que la propriété de stabilité par convolution. De cette propriété, nous déduisons la fonction de réflexion pour plusieurs tubes "en série», et pour un branchement de trois tubes. Une extension de ces formules au cas des tuyaux cylindriques fermés à parois rigides est donnée en Annexe A.
\end{abstract}

\begin{abstract}
As dissipation and dispersion of plane waves in a cylindrical tube are proportional to the square root of frequency, the form of the impulse response can be obtained analytically. We analyse the reflection function at the input of the tube, i.e. the inverse Fourier transform of the reflection coefficient, especially the important distortions due to a numerical treatment and the property of stability through convolution. Using this property, the reflection function is determined for a sequence of tubes as well as for a connection of three tubes. An extension of the formulas to the case of rigid-walled closed cylindrical tube is given in Appendix A.
\end{abstract}

\section{Introduction.}

The reflection function, i.e. the inverse Fourier transform of the reflection coefficient, is also the impulse response of a tube provided with a perfect absorber above its input. The interest of this function lies in the fact that it decays to zero much more rapidly than the traditional impulse response. This property leads Schumacher $[1,2]$ to develop oscillation models for wind instruments in which the resonator of the instrument.is represented by its reflection function. Some authors $[1,3,4]$ numerically calculated reflection functions for various shapes of tubes.

It is possible to analytically calculate the reflection function of a cylindrical tube. This allows one to assess the consequences of a numerical treatment on such a function, and study its properties.

After a review of the reflection coefficient calculation in a cylindrical tube, the form of the reflection functions is given and its properties discussed. Finally, some calculations based on the property of stability through convolution are developed.

\section{Calculation of the reflection function for a cylin-} drical tube open or closed.

In a cylindrical tube, the one-dimensional equations of the transmission line theory are (see e.g. [5]) :

$$
\partial_{x} p=-Z_{\mathrm{v}} \cdot u, \quad \partial_{x} u=-Y_{\mathrm{t}} \cdot p
$$

in which $Z_{\mathrm{v}}$ and $Y_{\mathrm{t}}$ are respectively the serie impedance per unit of length due to viscous effects and the shunt admittance per unit of length due to thermal effects, $p$ is the acoustic pressure and $u$ the particle velocity

$$
\begin{aligned}
& Z_{\mathrm{v}}=j \omega \rho\left(1+\frac{\sqrt{2}}{r_{\mathrm{v}}}(1-j)+0\left(\frac{1}{r_{\mathrm{v}}^{2}}\right)\right) \\
& Y_{\mathrm{t}}=\frac{j \omega}{\rho c^{2}}\left(1+\frac{\sqrt{2}}{r_{\mathrm{t}}}(1-j)(\gamma-1)+0\left(\frac{1}{r_{\mathrm{t}}^{2}}\right)\right)
\end{aligned}
$$

and

$$
\begin{aligned}
& r_{\mathrm{v}}=R\left[\frac{\omega}{c \cdot l_{\mathrm{v}}}\right]^{1 / 2} \\
& r_{\mathrm{t}}=R\left[\frac{\omega}{c \cdot l_{\mathrm{t}}}\right]^{1 / 2}
\end{aligned}
$$

$l_{\mathrm{v}}=\frac{\mu}{\rho c}$ is the viscous characteristic length, where $\mu$ is the first coefficient of viscosity,

$l_{\mathrm{t}}=\frac{\lambda}{\rho c C_{\mathrm{p}}}$ is the thermal characteristic length, where $\lambda$ is the coefficient of thermal conductivity (note that $l_{\mathrm{v}}$ and $l_{\mathrm{t}}$ have the same order of magnitude), $R \quad$ is the radius of the tube 
$\rho \quad$ is the density of air,

$c \quad$ is the speed of sound in free air,

$\omega=2 \pi f$ is the angular frequency,

$\gamma=C_{\mathrm{p}} / C_{\mathrm{v}}$ where $C_{\mathrm{p}}$ and $C_{\mathrm{v}}$ are the specific heats of air at constant pressure and constant $j=\sqrt{-1}$. volume, respectively,

Only the first order of visco-thermal effects will be taken into account. This assumption implies the following inequality : $R \gg\left(l_{\mathrm{v}} \lambda_{\mathrm{w}}\right)^{1 / 2}$, where $\lambda_{\mathrm{w}}$ is the wavelength. The radius of the tube also must not be too large compared to the wavelength for the assumption of plane wave to be satisfied.

The solution of equation (1) is :

$$
p(x)=\left(a \mathrm{e}^{-\Gamma x}+b \mathrm{e}^{\Gamma x}\right) \mathrm{e}^{j \omega t}
$$

where $a$ and $b$ are the complex amplitudes of outgoing and incoming waves respectively, and $\Gamma=\left(Z_{\mathrm{v}} \cdot Y_{\mathrm{t}}\right)^{1 / 2}$ is the propagation constant. The reflection coefficient $r(\omega)=b / a$ is then found to be :

$$
r(\omega)=\frac{Z(x)-\xi}{Z(x)+\xi} \mathrm{e}^{-2 \Gamma x}
$$

where $Z(x)$ is the impedance at the point $x$, i.e. the ratio $p(x) / u(x)$, and $\xi=\left(Z_{\mathrm{v}} / Y_{\mathrm{t}}\right)^{1 / 2}$ is the characteristic impedance of the tube.

Considering the origin $(x=0)$ at the input of the cylinder, the impedance at the output $x=l$ is given by the formulae :

$$
\begin{aligned}
& Z(l)=\infty \quad \text { if closed } \\
& Z(l)=\rho c\left[j \omega \Delta l / c+0\left(\omega^{2} R^{2} / c^{2}\right)\right] \text { if open }
\end{aligned}
$$

where

$$
\Delta l=0.6133 R \text {. }
$$

Here, only the reactive term of the formula given by Levine and Schwinger [7] for the radiation impedance is kept. Consequently, the dissipation due to radiation is considered negligible compared to the visco-thermal dissipation, as it is often the case.

The reflection coefficient at the input of the tube is

$$
\begin{aligned}
& r(\omega)=\mathrm{e}^{-2 \Gamma l \quad \text { if closed }} \\
& r(\omega)=-\mathrm{e}^{-2 \Gamma l-2 Z(l) / \xi+0\left(\omega^{2} R^{2} / c^{2}\right)}
\end{aligned}
$$

or

$$
r(\omega)=-\mathrm{e}^{-2 \Gamma l-2 \Gamma^{-} \Delta l} \quad \text { if open }
$$

and

$$
\begin{gathered}
\Gamma=\Gamma^{+}, \quad \Gamma^{ \pm}= \pm \sqrt{\frac{\omega}{2}} \frac{\alpha^{ \pm}}{R}(1+j)+j \frac{\omega}{c} \\
\alpha^{ \pm}=\left(\frac{1}{r_{\mathrm{v}}} \pm(\gamma-1) \frac{1}{r_{\mathrm{t}}}\right) \frac{\sqrt{\omega}}{c} R= \\
=\left(\sqrt{l_{\mathrm{v}}} \pm(\gamma-1) \sqrt{l_{\mathrm{t}}}\right) / \sqrt{c} .
\end{gathered}
$$

Dissipation is represented by the term $\sqrt{\frac{\omega}{2}} \frac{\alpha}{R}$, and dispersion by the term $j \sqrt{\frac{\omega}{2}} \frac{\alpha}{R}$.

The inverse Fourier transform $r(t)$ of $r(\omega)$, will obviously be stable through convolution because $(-2 \Gamma l)$ can be written as a linear combination of $\omega^{1 / 2}$ and $\omega, e \omega^{1 / 2}+d \omega$, where $e$ and $d$ are independent of frequency. Then,

$$
\mathrm{e}^{e_{0} \sqrt{\omega}+d_{0} \omega} \mathrm{e}^{e_{1} \sqrt{\omega}+d_{1} \omega}=\mathrm{e}^{\left(e_{0}+e_{1}\right) \sqrt{\omega}+\left(d_{0}+d_{1}\right) \omega} .
$$

The residue method leads to the inverse Fourier transform $r(t)$ :

$$
\begin{aligned}
& r(t)=\frac{A}{\sqrt{\pi}} f(t-\tau, A) \\
& f(t-\tau, A)=\frac{\mathrm{e}^{-A^{2} /(t-\tau)}}{(t-\tau)^{3 / 2}} \quad \text { for } t>\tau \\
& f(t-\tau, A)=0 \quad \text { for } t \leqslant \tau
\end{aligned}
$$

where $A=\alpha \frac{l}{r} ; \tau=\frac{2 l}{c}$ for the closed tube, and

$$
r(t)=-\frac{A}{\sqrt{\pi}} f(t-\tau, A)
$$

where $A=\alpha \frac{l}{r} ; \tau=\frac{2 l^{\prime}}{c}$ for the open tube (for simplicity, we put $\Gamma^{+} l+\Gamma^{-} \Delta l \approx \Gamma^{+} l^{\prime}$, where $l^{\prime}=l+\Delta l$, if $\Delta l \ll l$ ).

When positive, $r(t)$ is a well-known probability law [8].

We verify the good agreement of this result with the numerical calculation of $r(t)$ by inverse Fast Fourier Transform (FFT) of $r(\omega)$. The confrontation with experiment is made with a $25 \mathrm{~m}$ long tube of $4 \mathrm{~mm}$ radius, on which we insert a $1 / 8$ inch-microphone at a $10 \mathrm{~m}$ distance from the loudspeaker (see Fig. 1), so that we measure $r(t)$ for an open tube of $15 \mathrm{~m}$ long and $4 \mathrm{~mm}$ radius. This large value of the ratio $l / r$ allows us to partly avoid problems due to the numerical treatment, as it will be explained below.

The loudspeaker generates a small-duration transient signal picked-up by the microphone when going

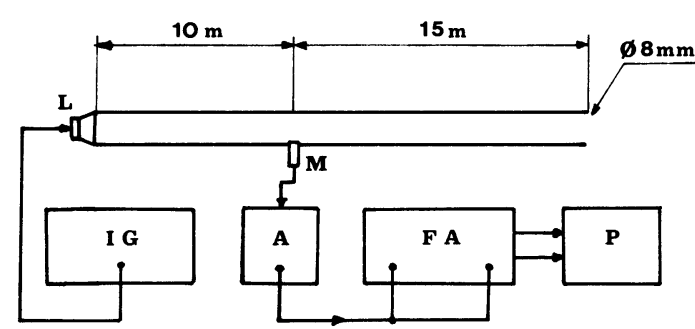

Fig. 1. - Measuring system for the reflection function of a tube of length $15 \mathrm{~m}$ and radius $4 \mathrm{~mm}$. L : Loudspeaker, M : Microphone, IG : Impulse Generator, A : Microphone Amplifier, FA : Fourier Analyser, P : Plotter. 
out (time $t=0$ at the arrival of the signal) and coming in, each signal being sent to a channel of the Fourier analyser. In order to avoid disturbances due to the measuring system, and to take into account the fact that the outgoing signal is not a Dirac impulse, we operate an inverse convolution of the incoming impulse (which has been filtered in order to take-off the high frequency noise) by the outgoing impulse. The result of this measurement is in good agreement with the analytic formula, as it can be seen in figure 2 . Slight differences are largely due to problem of time-domain windowing of the signals.

Appendix A gives results similar to equation (7) in the case of rigid-walled closed cylindrical tube, obtained by using another method.

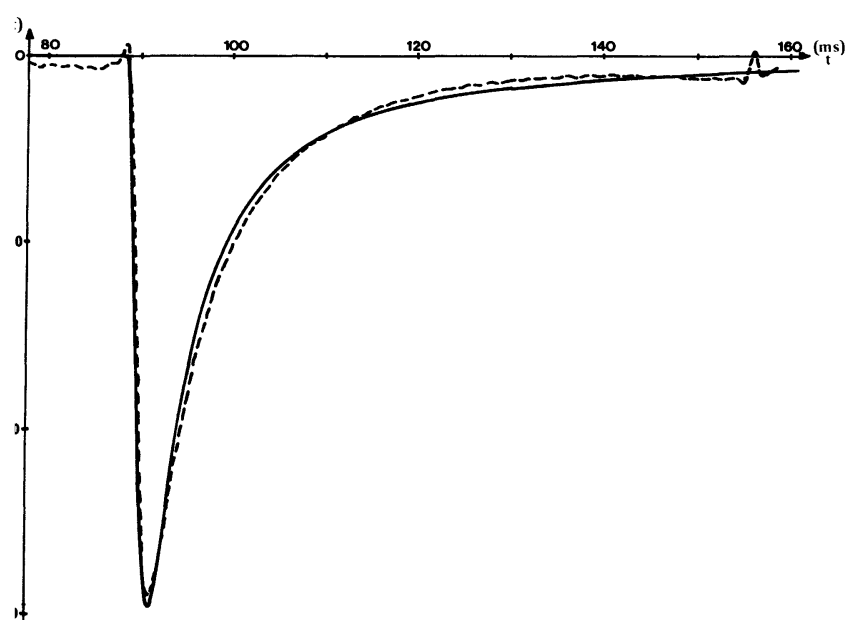

Fig. 2. - Reflection function (in $\mathrm{s}^{-1}$ ) of an open tube of length $15 \mathrm{~m}$ and radius $4 \mathrm{~mm}:$ Calculated (Eq. 7b), - - Experimental.

\section{Properties of $r(t)$; problems of numerical treat-} ment.

We can easily verify that $\left\|\int_{-\infty}^{+\infty} r(t) \mathrm{d} t\right\|=1$, due to the fact that the first order of visco-thermal effects is only considered. Therefore, the dissipation decreases to zero along with the frequency. The convergence of $r(t)$ is very slow, increasing with the ratio $l / r$. Figure 3 shows $\int_{0}^{t} r\left(t^{\prime}\right) \mathrm{d} t^{\prime}$ for various closed tubes.

If dissipation at very low fequencies had been taken into account, with the whole development of $Z_{\mathrm{v}}$ and $Y_{\mathrm{t}}$ (see Ref. [5]), the decay of $r(t)$ would be different from $t^{-3 / 2}$, particularly if the tube has a capillary behaviour. Nevertheless, the result remains valid as long as visco-thermal dissipation for the fundamental resonance frequency of the tube is properly represented by the values of $Z_{\mathrm{v}}$ and $Y_{\mathrm{t}}$ given in equations (2a) and (2b). As a matter of fact, calculation of the propagation constant $\Gamma$ at the

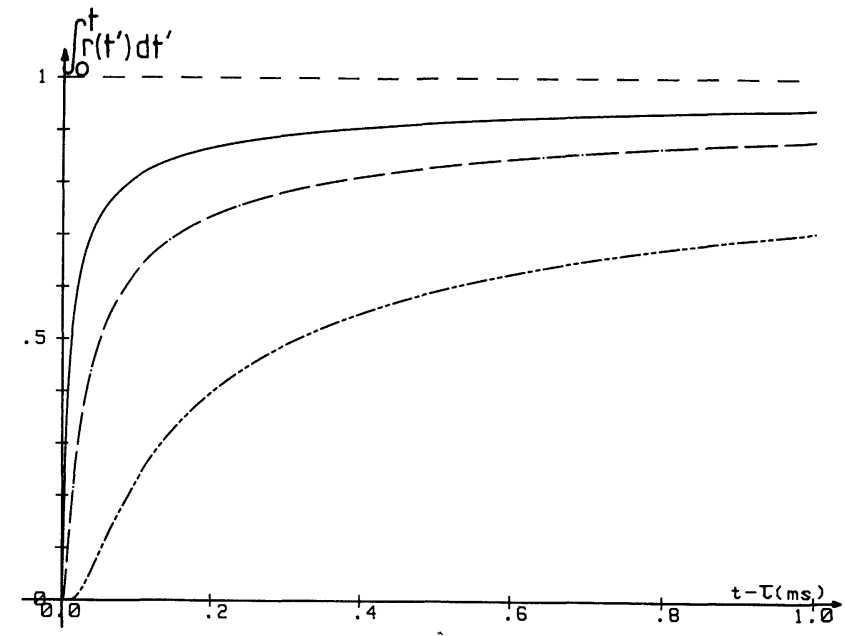

Fig. 3. - Convergence of the reflection function for three closed tubes of length $1 \mathrm{~m}$ and various radii : $0.2 \mathrm{~cm}$

second order of $1 / r_{\mathrm{v}}$ and $1 / r_{\mathrm{t}}$ shows that equation (7) is simply modified by multiplying the result by the factor : $\exp \left(-2 H l_{\mathrm{v}} l / R^{2}\right)$, where :

$$
\begin{aligned}
H=1+(\gamma-1)\left(l_{\mathrm{v}} / l_{\mathrm{t}}\right)^{1 / 2}- & \\
& -\gamma(\gamma-1) l_{\mathrm{t}} / 2 l_{\mathrm{v}} \# 1 \text { for air }
\end{aligned}
$$

(see e.g. Ref. [6]). Then a condition of validity for equation (7) is $l_{\mathrm{v}} l \ll R^{2}$.

The delay from $\tau$ to the time for which $\|r(t)\|$ is maximum is the following:

$$
t_{\max }=\frac{2}{3} A^{2}
$$

and

$$
r\left(\tau+t_{\max }\right)=\frac{1}{A^{2} \sqrt{\pi}}\left(\frac{3}{2}\right)^{3 / 2} \mathrm{e}^{-3 / 2} \simeq \frac{0.23}{A^{2}}
$$

$t_{\max }$ varies as $(l / r)^{2}$ and is generally very short, e.g. $t_{\max } \simeq 1.6 \mu \mathrm{s}$ for a tube (noted $\mathrm{T}$ ) of $1 \mathrm{~m}$ length and $1 \mathrm{~cm}$ radius.

So, we can see that for the calculation of $r(t)$ by the inverse FFT of $r(\omega) . F(\omega)$ where $F(\omega)$ is the frequency window of analysis, the disturbance introduced by the numerical treatment (Sampling, windowing, ...) is very large.

Thus, performing for the tube noted $\mathrm{T}$ an inverse FFT of $r(\omega) \cdot F(\omega)$, where $F(\omega)$ is a rectangular window from 0 to $10 \mathrm{kHz}$, we obtain one sample every $50 \mu$ s (about 30 times $t_{\max }$ ). In this case, the result of the convolution of $r(t)$ with the inverse Fourier transform of the window is characteristic of the window rather than $r(t)$. This is illustrated by figure 4 , and is most obvious when the maximum frequency of analysis is low. In order to approximate by numerical calculation the genuine form of $r(t)$ for the tube noted T one has to take one sample 


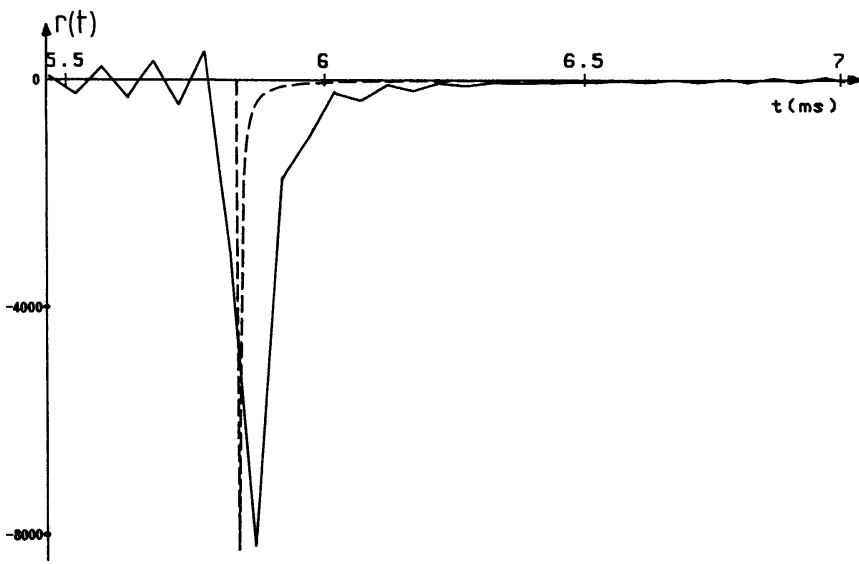

Fig. 4. - Comparison of reflection functions analytically and numerically calculated for an open tube of length $1 \mathrm{~m}$ and radius $1 \mathrm{~cm}$ : —- - Analytical, — inverse FFT (512 points, sampling frequency : $13.8 \mathrm{kHz}$, Maximum frequency of analysis : $5 \mathrm{kHz}$, Rectangular window).

every $t_{\max } / 5$, which implies a sampling frequency of 3.2 $\mathrm{MHz}$ and more than 20000 samples!

This function $r(t)$ is fairly different from the one used by Schumacher [2]. Consequently, the oscillation model gives appreciably different results, this being especially due to the symmetry of $r(t)$ around $t=\tau$ in the form chosen by Schumacher. We will discuss this question in future paper. Note that the equality of dissipation and dispersion terms implies the asymmetry of the curve of figure 2 .

\section{Self-convolution and applications.}

The stability through convolution of $r(t)$ allows us to write, if $r_{0}(t)$ corresponds to the couple $\left(R_{0}, l_{0}\right)$ and $r_{1}(t)$ to the couple $\left(R_{1}, l_{1}\right)$ :

$$
\begin{gathered}
r_{0}(t) * r_{1}(t)=\frac{A}{\sqrt{\pi}} f(t-\tau, A) \\
A=\alpha\left(\frac{l_{0}}{R_{0}}+\frac{l_{1}}{R_{1}}\right) \\
\tau=\frac{2}{c}\left(l_{0}+l_{1}\right) .
\end{gathered}
$$

where

This convolution, when multiplied by a certain factor which depends on the reflection and transmission between tubes, corresponds to the return trip of two tubes in « series ».

Let us consider an abrupt change in cross-section between two cylinders $T_{0}$ and $T_{1}$, with sections $S_{0}$ and $S_{1}$ respectively. By assuming the continuity of pressure (ignoring the acoustic mass the effect of which is only appreciable at high frequencies) and volume velocity on both sides of the change in crosssection, the reflection coefficient of the tube $\mathrm{T}_{0}$ near the discontinuity is $\left(S_{0}-S_{1}\right) /\left(S_{0}+S_{1}\right)$ and the transmission coefficient from tube $\mathrm{T}_{0}$ to tube $\mathrm{T}_{1}$ is $2 S_{1} /\left(S_{0}+S_{1}\right)$. So, according to the notation of

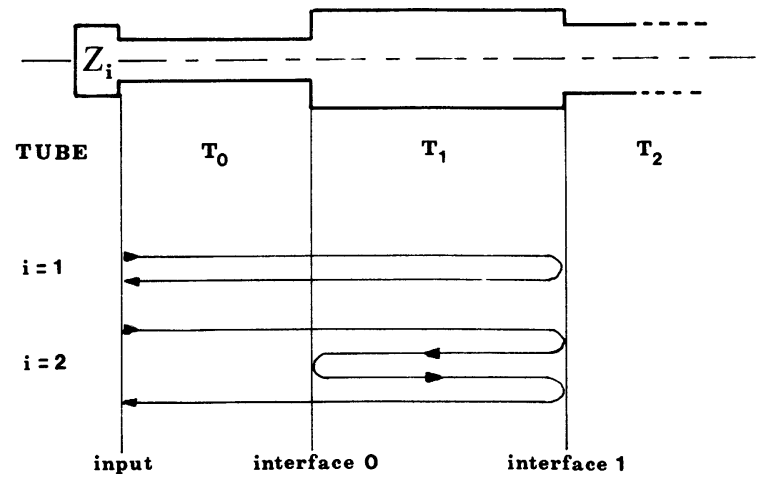

Fig. 5. - Tubes in « series ». Tube $\mathrm{T}_{0}$ : length $l_{0}$, radius $r_{0}$. Tube $\mathrm{T}_{1}$ : length $l_{1}$, radius $r_{1}$. Tube 2 : infinite length, radius $r_{2} . Z_{i} \simeq \rho c / S_{0}$ corresponds to a perfect absorber for tube $\mathrm{T}_{0} . i$ corresponds to the number of return trips performed by a given wave in tube $T_{1}$.

figure 5, the contribution to $r(t)$ from to a wave having travelled one time through $\mathrm{T}_{0}$ and $i$ times through $T_{1}$ is :

$$
r_{i}(t)=r_{0}(t) \cdot t_{01} *\left[r_{1}(t) \cdot r_{11} \cdot r_{10}\right]^{* i} \cdot \frac{1}{r_{10}} \cdot t_{10}
$$

where

$t_{01}=\frac{2 S_{1}}{S_{0}+S_{1}} \quad$ is the transmission coefficient from tube $T_{0}$ to tube $T_{1}$.

$t_{10}=\frac{2 S_{0}}{S_{0}+S_{1}} \quad$ is the transmission coefficient from tube $T_{1}$ to tube $T_{0}$.

$r_{11}=\frac{S_{1}-S_{2}}{S_{1}+S_{2}} \quad$ is the reflection coefficient of tube $\mathrm{T}_{1}$ at interface 1 .

$r_{10}=\frac{S_{1}-S_{0}}{S_{1}+S_{0}} \quad$ is the reflection coefficient of tube $\mathrm{T}_{1}$ at interface 0 .

$r_{0}(t)$ and $r_{1}(t)$ respectively are the reflection functions of tubes $T_{0}$ and $T_{1}$ when they are closed

the exponent $*_{i}$ is the self-convolution repeated $i$ times.

One can easily deduce the form of the reflection function at the input of tube $T_{0}$ :

$$
r(t)=g_{0}(t) *\left\{\delta(t)+T_{01} \sum_{i=1}^{\infty}\left(g_{1}(t)\right)^{* i}\right\}
$$

where

$$
\begin{gathered}
g_{0}(t)=\frac{A_{0}}{\sqrt{\pi}} f\left(t-\tau_{0}, A_{0}\right) \cdot \frac{S_{0}-S_{1}}{S_{0}+S_{1}}, \\
A_{0}=\alpha \frac{l_{0}}{R_{0}} \quad \tau_{0}=\frac{2 l_{0}}{c} \\
g_{1}(t)=\frac{A_{1}}{\sqrt{\pi}} f\left(t-\tau_{1}, A_{1}\right) \cdot \frac{S_{1}-S_{2}}{S_{1}+S_{2}} \cdot \frac{S_{1}-S_{0}}{S_{1}+S_{0}}, \\
A_{1}=\alpha \frac{l_{1}}{R_{1}} \quad \tau_{1}=\frac{2 l_{1}}{c}
\end{gathered}
$$




$$
T_{01}=-\frac{4 S_{0} S_{1}}{\left(S_{0}-S_{1}\right)^{2}}, \quad S_{0}=\pi r_{0}^{2}, \quad S_{1}=\pi r_{1}^{2}
$$

$\delta(t)$ is the Dirac-impulse.

Of course, $\sum_{i=1}^{\infty}\left(g_{1}(t)\right)^{* i}$ is the impulse response of tube $T_{1}$ when connected at the output from the tube of radius $R_{2}$, and at the input to a tube of radius .$R_{0}$.

After calculation of the convolutions, we get :

$$
\begin{gathered}
r(t)=\frac{S_{0}-S_{1}}{S_{0}+S_{1}} \cdot \frac{A_{0}}{\sqrt{\pi}} f\left(t-\tau_{0}, A_{0}\right)- \\
-\frac{4 S_{0} S_{1}}{S_{0}^{2}-S_{1}^{2}} \sum_{i=1}^{\infty} \frac{A_{i}}{\sqrt{\pi}} f\left(t-\tau_{i}, A_{i}\right) \beta^{i}
\end{gathered}
$$

where

$$
\begin{gathered}
A_{i}=\alpha\left(\frac{l_{0}}{R_{0}}+i \frac{l_{1}}{r_{1}}\right), \quad \tau_{i}=\frac{2}{c}\left(l_{0}+i l_{1}\right) \\
\beta=\frac{S_{1}-S_{0}}{S_{1}+S_{0}} \cdot \frac{S_{1}-S_{2}}{S_{1}+S_{2}} .
\end{gathered}
$$

In a general way, when we connect a tube $T_{n}$ in series to tube $T_{n-1}$, the new $r(t)$ at the input of tube $\mathrm{T}_{0}$ is obtained by replacing $\sum_{i=1}^{\infty}\left(g_{n-1}(t)^{* i}\right)$ with : $\sum_{i=1}^{\infty}\left[g_{n-1}(t)^{* i} *\left\{\delta(t)+T_{n-1, n} \sum_{j=1}^{\infty}\left(g_{n}(t)\right)^{* j}\right\}\right]$

where

$$
\begin{gathered}
T_{n-1, n}=-\frac{4 S_{n-1} \cdot S_{n}}{\left(S_{n-1}-S_{n}\right)^{2}} \\
g_{n}(t)=\frac{A_{n}}{\sqrt{\pi}} f\left(t-\tau_{n}, A_{n}\right) \cdot \frac{S_{n}-S_{n+1}}{S_{n}+S_{n+1}} \cdot \frac{S_{n}-S_{n-1}}{S_{n}+S_{n-1}} \\
A_{n}=\alpha \frac{l_{n}}{R_{n}}, \quad \tau_{n}=2 \frac{l_{n}}{c}
\end{gathered}
$$

in the expression of $r(t)$ for $n-1$ tubes (same form as Eq. (10)). The form of the reflection function at the input of tube $T_{0}$ corresponding to the case of figure 6 is :

$$
r(t)=\sum_{\left(i_{1}, i_{2}\right)=(0,0)}^{(\infty, \infty)} C_{i_{1} i_{2}} r_{i_{1} i_{2}}(t)
$$

where

$$
r_{i_{1} i_{2}}(t)=\frac{A_{i_{1} i_{2}}}{\sqrt{\pi}} f\left(t-\tau_{i_{1} i_{2}}, A_{i_{1} i_{2}}\right)
$$

and

$$
\begin{aligned}
A_{i_{1} i_{2}} & =\alpha\left(\frac{l_{0}}{R_{0}}+i_{1} \frac{l_{1}}{R_{1}}+i_{2} \frac{l_{2}}{R_{2}}\right) ; \\
\tau_{i_{1} i_{2}} & =\frac{2}{c}\left(l_{0}+i_{1} l_{1}+i_{2} l_{2}\right)
\end{aligned}
$$

$i_{1}$ and $i_{2}$ are respectively the numbers of return trips performed by the wave in tube $T_{1}$ and tube REVUE DE PHYSIQUE APPLIQUÉE. - T. 22, N 5, MAI 1987

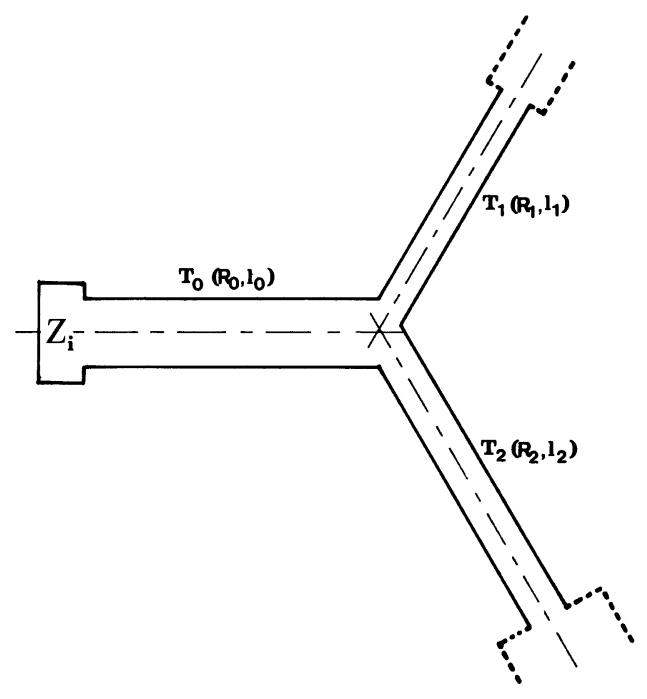

Fig. 6. - Three branched tubes. Tube $\mathrm{T}_{0}$ : length $l_{0}$, radius $r_{0}$. Tube $\mathrm{T}_{1}$ : length $l_{1}$, radius $r_{1}$. Tube $\mathrm{T}_{2}$ : length $l_{2}$, radius $r_{2} . Z_{i} \simeq \rho / S_{0}$ corresponds to a perfect absorber for tube $T_{0}$.

$\mathrm{T}_{2}$ respectively ; $C_{i_{1} i_{2}}$ is the coefficient resulting of all reflections and transmissions of all waves having performed $i_{1}$ round trips through tube $\mathrm{T}_{1}$ and $i_{2}$ round trips through tube $T_{2}$. Note that in general $\left\|\int_{-\infty}^{+\infty} r(t) \mathrm{d} t\right\|=1$, because the input impedance of low dissipative tubes of any shape is either capacitive (closed tubes) or inductive (open tubes), and $\|r(\omega)\|$ goes to unity when $\omega$ goes to zero (see Eq. (4)).

\section{Conclusion.}

We summarize the two main properties of the reflection function $r(t)$ for cylindrical tube :

- The shape of the curve of $r(t)$ is highly asymmetrical : its decay is very slow. Therefore, the oscillation symmetry obtained by Schumacher [2] is no longer valid, transients becoming steeper at their beginning than at their end ;

$-r(t)$ is stable through convolution, thus allowing very simple and fast calculations for elementary combinations of tubes.

Of course, the results are similar for any cylindrical tubes, whatever the shape of the cross section, but for large open tubes the dissipation due to radiation would be not negligible compared to visco-thermal losses. Note that the range of validity of our calculations corresponds to tubes well described at their first resonance by classical theory of viscothermal effects.

\section{Appendix A.}

PRESSURE WAVE FROM AN EXPLOSIVE SOURCE AT ONE END OF A RIGID-WALLED CYLINDRICAL CLOSED TUBE. - The aim of this Appendix is to show that 
the pressure wave from an explosive source at the input $(x=0)$ of a rigid-walled cylindrical closed tube presents the same behaviour as the one obtained in section 2 , if the acoustic response of the tube is calculated using the modal theory.

Any damping mechanism attenuates transients. If weak damping mechanisms are taken into account, we can expect the solution given by a three dimensional modal theory (which assumes small admittances at the boundaries) to be correct, even for terms corresponding to high order echoes which may have suffered large attenuations and phase shifts.

Let the piston deplacement at the input $x=0$ be the step function $H\left(t-t_{0}\right)$. The acoustic pressure generated by the source can be written as follows :

$$
p(\mathbf{r}, t)=\rho S \partial_{\mathrm{t}} G(\mathbf{r}, t)
$$

where $G(\mathbf{r}, t)$ is the time-dependent Green function (see for example [9, 10]), which is, disregarding the second-order terms with respect to $\varepsilon_{\mathrm{vt}}$ :

$$
\begin{aligned}
G(\mathbf{r}, t)=\frac{c}{2 \pi R L} \sum_{n=0}^{\infty} & {\left[\mathrm{e}^{-(n \pi / L)^{1 / 2} \varepsilon_{\mathrm{vt}} x^{-}} \frac{\sin \left(\alpha_{n} x^{-}\right)}{\alpha_{n}}+\right.} \\
& \left.+\mathrm{e}^{-(n \pi / L)^{1 / 2} \varepsilon_{\mathrm{vt}} x^{+}} \frac{\sin \left(\alpha_{n} x^{+}\right)}{\alpha_{n}}\right]
\end{aligned}
$$

with

$$
\begin{aligned}
x^{ \pm} & =c t \pm x \\
\varepsilon_{\mathrm{vt}} & =\frac{1}{\sqrt{2} R}\left[\sqrt{l_{\mathrm{v}}}+(\gamma-1)\left(1+\frac{2 R}{L}\right) \sqrt{l_{\mathrm{t}}}\right] \\
\alpha_{n} & =\frac{n \pi}{L}-(n \pi / L)^{1 / 2} \varepsilon_{\mathrm{vt}} .
\end{aligned}
$$

In this expression for $G(\mathbf{r}, t)$, the only terms introduced in the eigenfunction expansion are those which correspond to axial modes. Besides, in order to take into account the losses in the boundary layers at the walls, we assigned appropriate specific acoustic admittances for each mode and each wall in the cavity, including the walls at $x=0$ and $x=L$ (factor $2 R / L$ in the expression of $\varepsilon_{\mathrm{vt}}$ ).

The acoustic pressure can be taken as a superposition of left and right travelling waves, i.e.,

$$
p=p^{-}+p^{+}
$$

with

$$
\begin{aligned}
p^{ \pm}= & 2 p_{0} \sum_{n=0}^{\infty} \mathrm{e}^{-(n \pi / L)^{1 / 2} \varepsilon_{\mathrm{vt}} x^{ \pm}} \\
& \times \cos \left[\frac{n \pi}{L}-\left(\frac{n \pi}{L}\right)^{1 / 2} \varepsilon_{\mathrm{vt}}\right] x^{ \pm} \\
= & p_{0} \sum_{n=-\infty}^{\infty} \mathrm{e}^{-(2 \pi / L)^{1 / 2} \varepsilon_{\mathrm{vt}} x^{ \pm} \sqrt{j n}} \mathrm{e}^{j n \pi x^{ \pm} / L}
\end{aligned}
$$

where we neglected the term corresponding to the derivative with respect to $« t »$ of $\mathrm{e}^{-(n \pi / L)^{1 / 2} \varepsilon_{\mathrm{vt}} x^{ \pm}}$.

The indicated sum on « $n$ » can be calculated by assuming that $p^{ \pm}$is different from zero only for $x^{ \pm} \simeq 2 q L+c \delta t$, where $q$ is an integer and $\delta t$ an arbitrary short time $(\|\delta t\| \ll L / c)$. Without losses, the pulse would be located at $x^{ \pm}=2 q L$

We calculate $p^{-}$only ; the calculation of $p^{+}$can be carried out in the same way.

A common technique for calculating such an expression is to replace the sum by an integral so that we write :

$$
\begin{aligned}
\frac{p^{-}}{p_{0}}= & -\sum_{q=0}^{\infty} \sum_{n=-\infty}^{\infty} \mathrm{e}^{-(2 \pi / L)^{1 / 2} \varepsilon_{\mathrm{vt}}(2 q L+c \delta t) \sqrt{j n}} \\
& \times \mathrm{e}^{j n \pi c \delta t / L} \\
= & -\sum_{q=0}^{\infty} \int_{-j \infty}^{j \infty} \mathrm{e}^{-(2 \pi / L)^{1 / 2} \varepsilon_{\mathrm{vt}}(2 q L+c \delta t) \sqrt{j \nu}} \\
& \times \mathrm{e}^{j n \pi c \delta t / L} \mathrm{~d}(j \nu) .
\end{aligned}
$$

Note that the lower limit $q=0$ corresponds to the primary wave. For the other wave $p^{+}$, the lower limit is $q=1$. Besides, the various terms in the above sum remain null until $t$ is sufficiently large for their arguments $\left(c t \pm x-\frac{2 q L}{c}\right)$ to be positive. Thus there are only a finite number of nonzero terms in the sum for any finite value of $t$.

The primary wave $(q=0)$ being not considered in the next equations, the term $c \delta t$ is discarded in the expression $(2 q L+c \delta t)$. Then the results given in section 2 allow us to write :

$$
\begin{aligned}
\frac{p^{-}}{p_{0}}=\sum_{q=1}^{\infty} \frac{2 L}{c} \frac{\sqrt{2 / c} \varepsilon_{\mathrm{vt}} q L}{\sqrt{\pi}} & \times \\
& \times \frac{1}{(\delta t)^{3 / 2}} \mathrm{e}^{-\left[(2 / c)^{1 / 2} \varepsilon_{\mathrm{vt}} q L\right]^{2} / \delta t} .
\end{aligned}
$$

At the extremity $x=0$, we have $\delta t=t-$ $2 q L / c=t-q \tau$ (see Eq. (7)). Moreover knowing that $\alpha / R$ (Sect. 2) is equal to $(2 / c)^{1 / 2} \varepsilon_{\mathrm{vt}}$ and following the property given in section 4 (Eq. (9)), we can write (with $\left.A=(2 / c)^{1 / 2} \varepsilon_{\mathrm{vt}} L\right)$ :

$$
\frac{p^{-}}{T p_{0}}=\sum_{q=1}^{\infty} \frac{q A}{\sqrt{\pi}} \frac{\mathrm{e}^{-q^{2} A^{2} /(t-q \tau)}}{(t-q \tau)^{3 / 2}}
$$

where $T$ is the period, equal to the round-trip time $2 L / c$ (note that $r(t)$ in Eq. (7) is homogeneous to $\left.s^{-1}\right)$.

This result emphasizes that each term in the sum has exactly the same form as the result given in equation (7a). 


\section{References}

[1] Schumacher, R. T., Acustica 48 (1981) 71.

[2] MC INTYRE, M. E., SCHUMACHER, R. T., Woodhouse, J., J. Acoust. Soc. Am. 74 (1983) 1325.

[3] Agullo, J., Barjau, A., J. Sound Vib. 106 (1986) 193.

[4] Ayers, R. D., Eliason, L. J., 12th International Congress on acoustics (Toronto, July 1986) K 45.

[5] Zwikker, C., Kosten, C., Sound Absorbing Materials (Elsevier, Amsterdam) 1949.

[6] Kergomard, J., J. Sound Vib. 98 (1985) 149.
[7] Levine, H., Schwinger, J., Phys. Rev. 73 (1948) 383.

[8] Koroliouk, V., Aide Mémoire de Théorie des Probabilités et de Statistiques Mathématiques, e.g. see Chapter on Stable laws of probability, p. 137 (Eds. de Moscou) 1983.

[9] BRUNEAU, M., Introduction aux théories de l'acoustique (Ed. de l'Université du Maine, Le Mans) 1983, p. 321.

[10] Pierce, A. D., Acoustics, an introduction to its physical principles and applications (McGraw Hill, New York) 1983. 